\title{
The Effect of the Solvent on the Base Catalysed Conjugation Isomerization of Poly-unsaturated Fatty Acid Compounds
}

\author{
JOHN UGELSTAD, ODDA. ROKSTAD and JAN SKARSTEIN
}

Institute of Industrial Chemistry, Norges Tekniske Högskole, Trondheim, Norway

\begin{abstract}
The conjugation isomerization of linseed oil methyl ester catalysed by $\mathrm{K}$ - $t$-butylate has been investigated with ether and with mixtures of ether and dimethyl formamide, ethylene glycol dimethyl ether, diethylene glycol dimethyl ether, and tetraethylene glycol dimethyl ether as solvents. The rates of the reactions in the pure ether solution are much higher than those observed when $t$-butanol is used as solvent. A marked increase in the rate was observed when a small amount of the ether was replaced by any of the above substances. When com. pared at the same percentage by volume of the additive the accelerating effect increased markedly with increasing chain length of the glycol ether. Thus, at a conc. of $1 \%(v / v)$, the effect of diethylene glycol dimethyl ether on the rate was slightly less than the effect of dimethyl formamide, while the effect of tetraethylene glycol dimethyl ether was about 8 times greater than that of dimethyl formamide. With $2 \%$ tetraethylene glycol dimethyl ether the first step in the conjugation isomerization was completed in about $3 \mathrm{~min}$ at $25^{\circ} \mathrm{C}$.
\end{abstract}

The rate of the conjugation isomerization of poly-unsaturated fatty acids 1 in butanol with $\mathrm{K}$ - $n$-butylate as catalyst has been shown to increase enormously if the alcohol is partly replaced by dimethyl sulfoxide, dimethyl formamide, or tetramethyl urea ${ }^{1}$. The effect was attributed to an increase in the $\mathrm{RO}^{-}$activity resulting from: (1) The ability of dimethyl sulfoxide etc. to bind the alcohol by $\mathrm{H}$-bonds, thus reducing the deactivating effect of alcohol on the alcoholate ion by $\mathrm{H}$-bond formation $\mathrm{RO}-\mathrm{H}$. . - OR. (2) The ability of dimethyl sulfoxide etc. to solvate the $\mathrm{K}^{+}$ion specifically, thus rendering the $\mathrm{RO}^{-}$ion in a more active state than it would be in an "inert" solvent.

In the present work the conjugation isomerization of linseed oil methyl ester has been carried out in ether solution with $\mathrm{K}$-t-butylate as catalyst, and the influence of various substances on the rate of the conjugation isomerization has been investigated. As no alcohol is present, the specific solvation of the $\mathrm{K}^{+}$ion would be expected to be the only effect.

Zaugg ${ }^{2}$ measured the effect of various additives on the rate of the alkylation of sodio-malonic esters in benzene solution, and suggested that for a 
substance to be able to give a marked increase in the solvation of the $\mathrm{Na}^{+}$ ion it must contain an $\mathrm{O}$-atom with a high electron density in the $\pi$-orbital at the $\mathrm{O}$-atom. Such is claimed to be the case for dimethyl sulfoxide, dimethyl formamide, and tetramethyl urea, all of which Zaugg found had a considerable accelerating effect on the rate of the alkylation. However, there are several observations in the literature which indicate that a considerable difference in the capacity of alkali-ion solvation may be present even for substances where Zaugg's explanation is not applicable. Thus both tetrahydrofuran and ethylene glycol dimethyl ether were found to give an increase in the rate of the alkylation reaction, although the effect of these substances were much smaller than the effect of the "very active" substances ${ }^{2}$. Scott et al. ${ }^{3}$ found that the reaction $\mathrm{Na}+$ naphtalene $\rightarrow \mathrm{Na}^{+}+$naphtalene ${ }^{-}$takes place much more readily in dimethyl ether and ethylene glycol dimethyl ether than in diethyl ether. Zook and Russo ${ }^{4}$ reported that the alkylation of sodio-butyrophenone proceeded much faster in dimethyl ethers of glycol and diethylene glycol than in diethyl ether. In both cases the effects are ascribed to an increased solvation of the $\mathrm{Na}^{+}$ion. During the investigation of the effect of different additives on the rate of the conjugation isomerization in diethyl ether solution the effect of ethylene glycol dimethyl ether and some higher homologues as well as the effect of dimethyl formamide was therefore measured.

\section{EXPERIMENTAL}

$$
\text { Procedure }
$$

To a relatively concentrated solution of $\mathrm{K}$ - $t$-butylate in ether various amounts of ether and additives were added so that the catalyst solution in every case contained $0.4 \mathrm{~mole} / \mathrm{l}$ of $\mathrm{K}$ - $t$-butylate. The isomerization reactions were carried out in $25 \mathrm{ml}$ Erlenmeyer flasks fitted with glass stoppers. Half way up the wall, the flask was directly connected to a closed end glass tube, which was sealed to the flask by fusing. A micro beaker containing the weighed sample of linseed oil was placed in the tube; the catalyst solution was placed in the bottom of the flask. The temperature of the flask was adjusted to $25^{\circ} \mathrm{C}$ in a thermostat. At time zero the vessel containing the linseed oil was tipped into the catalyst solution, and the flask was shaken vigorously. After a given time the reaction was stopped by addition of methanol. The whole procedure involving the preparation of the catalyst solution and the isomerization process was carried out in a $\mathrm{N}_{2}$-atmosphere. In each run $10 \mathrm{ml}$ of catalyst solution was used, the amount of the linseed oil methyl ester varied between 60 and $240 \mathrm{mg}$. The specific extinction coefficients for conjugated tri-ene $(267 \mathrm{~m} \mu)$ and di-ene $(234 \mathrm{~m} \mu)$ were measured with a Beckman spectrophotometer, model DU.

\section{$\mathrm{R}$ e a g e n t s}

Linseed oil methyl ester. The product made from commercial linseed oil was refined by molecular distillation.

Diethyl ether. Commercial diethyl ether free from peroxides was dried over sodium and distilled.

Potassium-t-butylate. $21 \mathrm{~g}$ of potassium (Riedel de Haën A. G.) and $40 \mathrm{ml}$ xylene (E. Merck A.G.) was heated in a 1 liter round-bottom flask until the rim of boiling xylene reached the middle of the flask. Then a stopper was inserted, the flask shaken five or six times and allowed to cool. Xylene was decanted and the finely divided metal was washed three times with absolute ether by decantation. Then about $500 \mathrm{ml}$ absolute ether was poured into the flask, a condenser provided with a calcium chloride tube was attached to the flask and $34 \mathrm{~g}$ of $t$-butanol (E. Merck A.G.) slowly added through a separatory

Acta Chem. Scand. 17 (1963) No. 1 
funnel. After one hour a Bunsen valve was attached at the top of the system. To complete the reaction the mixture was refluxed for $24 \mathrm{~h}$. Insoluble materials were then allowed to settle, and the liquid solution decanted. The concentration of $\mathbf{K}$-t-butylate determined by titration was $\sim 0.9$ mole/l.

$N, N$-dimethyl formamide of "for ultraviolet spectroscopy" grade was obtained from E. Merck A.G.

Ethylene glycol dimethyl ether. The "purum" quality (99\%) from Fluka A.G. contained some water and peroxides. Peroxides were removed by reduction with sodium and small amounts of water. The ether was then dried over sodium. The decanted liquid showed no base content.

Diethylene glycol dimethyl ether. Peroxides and water were removed from the pract. grade $(95 \%$ ) reagent from Fluka A.G. as described above. The decanted liquid contained base, and was therefore fractionally distilled under reduced pressure in an atmosphere of nitrogen.

Tetraethylene glycol dimethyl ether. The "purum" grade reagent from Fluka A.G. contained no peroxides. It was dried with sodium and then fractionally distilled under reduced pressure in an atmosphere of nitrogen.

\section{RESULTS}

Table 1 and 2 give the results of the kinetic measurements carried out in pure ether solutions with varying amounts of linseed oil methyl ester.

Table 1. Specific extinction coefficients $(1 / \mathrm{g} \mathrm{cm})$ at $267 \mathrm{~m} \mu$ after different time intervals (minutes). Catalyst solution: $10 \mathrm{ml}$ ether containing $0.4 \mathrm{~mole} / 1 \mathrm{~K}$-t-butylate. Temp. $25^{\circ} \mathrm{C}$.

\begin{tabular}{|c|c|c|c|c|c|c|c|c|c|c|c|}
\hline $\begin{array}{l}\text { Linseed oil } \\
\text { methyl ester }\end{array}$ & 5 & 10 & 20 & 30 & 45 & 60 & 90 & 120 & 180 & 240 & 300 \\
\hline $60 \mathrm{~m}$ & 2.1 & 4.7 & 10.2 & 15.1 & 21.5 & 25.9 & 32.2 & 35.8 & 39.3 & 41.0 & 41.5 \\
\hline $120 \mathrm{mg}$ & & 4.4 & 9.9 & 14.8 & 21.4 & 26.1 & 32 & 36.7 & 40.3 & 41.0 & 41.8 \\
\hline $240 \mathrm{mg}$ & & 5.1 & 10.5 & 15.8 & 22.4 & 27.0 & 33.5 & 37.1 & 41.5 & 42.6 & 43.0 \\
\hline
\end{tabular}

Table 2. Specific extinction coefficients at $234 \mathrm{~m} \mu$. Conditions as for Table 1 .

\begin{tabular}{|c|c|c|c|c|c|c|c|c|c|c|c|}
\hline $\begin{array}{c}\text { Linseed oil } \\
\text { methyl ester }\end{array}$ & 5 & 10 & 20 & 30 & 45 & 60 & 90 & 120 & 180 & 240 & 300 \\
\hline & & & & & & & & & & & \\
$60 \mathrm{mg}$ & 5.6 & 9.3 & 14.9 & 19.4 & 25.0 & 28.9 & 35.1 & 38.0 & 42.6 & 45.1 & 46.1 \\
$120 \mathrm{mg}$ & & 8.9 & 14.8 & 19.5 & $\mathbf{2 5 . 4}$ & $\mathbf{2 9 . 6}$ & $\mathbf{3 5 . 7}$ & $\mathbf{3 9 . 7}$ & $\mathbf{4 3 . 4}$ & $\mathbf{4 5 . 7}$ & $\mathbf{4 6 . 8}$ \\
$240 \mathrm{mg}$ & & 9.5 & & 20.0 & $\mathbf{2 5 . 8}$ & 29.9 & $\mathbf{3 5 . 6}$ & $\mathbf{3 9 . 3}$ & $\mathbf{4 4 . 0}$ & $\mathbf{4 6 . 3}$ & $\mathbf{4 7 . 4}$ \\
\hline
\end{tabular}

As will be seen from the tables, the specific extinction coefficients obtained at a particular reaction time are independent of the amount of the linseed oil, indicating that the reactions are first order with respect to the linseed oil. The specific extinction coefficients approach apparently constant values after a reaction time of about $4 \mathrm{~h}$. The rate in pure ether solution is remarkably fast compared to the rate observed with $t$-butanol as solvent. Sreenivasan and Brown ${ }^{5}$ investigated the rate of the conjugation of linolic acid and linolenic acid concentrates in $t$-butanol with $\mathrm{K}$ - $t$-butylate as catalyst, and found 
that with approximately 0.5 mole $/ 1 \mathrm{~K}$ - $t$-butylate it took nearly $8 \mathrm{~h}$ at $84^{\circ} \mathrm{C}$ to reach a degree of conjugation corresponding to that obtained after $4 \mathrm{~h}$ at $25^{\circ} \mathrm{C}$ in the present case. The much higher rate observed in ether solution is probably due to the absence of the alcohol, which has a considerable deactivating effect. Moreover, the ether as discussed below probably also leads to an activation of the catalyst by interaction with the $\mathbf{K}^{+}$ion.

Table 3 gives the relative rates observed when some of the ether is replaced by various other substances. Only relatively small amounts of the most "active" additives have been used as otherwise the rates became too high to be measured accurately. By increasing the amounts of the most active additives there was a slight shift in the "end-values" of the extinction coefficients, a decrease in the tri-ene value and an increase in the di-ene value. The relative rates given in Table 3 are calculated from a comparison of the time necessary to give a specific extinction coefficient of 15 at $267 \mathrm{~m} \mu$.

Table 3. Rates of formation of conjugated tri-ene, relative to the rate in diethyl ether. The amount of substance added is given as per cent of the volume of the catalyst solution. Temp. $25^{\circ} \mathrm{C}$. (K-t-butylate) $=0.4 \mathrm{~mole} / \mathrm{l}$.

\begin{tabular}{|l|c|r|r|r|r|r|r|}
\hline Substance added, \% & 0.5 & 1.0 & 1.5 & 2.0 & 3.0 & 4.0 & 5.0 \\
\hline $\begin{array}{l}\text { Monoethylene glycol } \\
\text { dimethyl ether }\end{array}$ & & & & & & & \\
$\begin{array}{l}\text { Diethylene glycol } \\
\text { dimethyl ether } \\
\begin{array}{l}\text { Tetraethylene glycol } \\
\text { dimethyl ether } \\
\text { N,N-Dimethyl formamide }\end{array}\end{array}$ & 20 & 40 & 62 & 86 & 3.2 & 4.1 & 5.2 \\
\hline
\end{tabular}

It may be seen from Table 3 that dimethyl formamide, even in small amounts, leads to a marked increase in the rate of the conjugation isomerization. The accelerating effect of the ethylene glycol dimethyl ether is also clearly noticeable, although it is much smaller. However, by increasing the chain length of the glycol ether the accelerating effect is remarkably increased. When compared at a concentration of $1 \%$ by volume, the effect of diethylene glycol dimethyl ether on the rate is about the same as that of dimethyl formamide, whilst the effect of tetraethylene glycol dimethyl ether is about 8 times greater than that of dimethyl formamide. The increase in the accelerating effect of the glycol ether with increasing chain length would be even greater if compared at the same molar concentration. However, it is apparent from the results given in Table 3 that the "order" of the reaction with respect to the additive is greater with dimethyl formamide than with the poly-glycol ethers.

\section{DISCUSSION}

Zaugg ${ }^{2}$ found that there seems to be no correlation between the total dipole moment of the additives and their ability to solvate the alkali ion as would be postulated by older theories of ion-dipole interaction. Zaugg proposes, 
however, that a necessary condition for a substance to be very effective in this respect and thus to have an outstanding catalytic efficiency is that it has a high $\pi$-moment. As is evident from our results, it seems that such a restriction sets a too narrow limit to possible active additives. In fact our results indicate that for reactions where the reactivity of the anion depends upon the solvation of the alkali-ion, tetraethylene glycol dimethyl ether may compete favourably with any of the active additives cited by Zaugg.

There seems to be a correlation between the capacity of a substance with a single active $\mathrm{O}$-atom for solvating alkali ions and its capacity to act as an "acceptor" in the formation of H-bonds. Nor in this case is there a correlation between the dipole moment of the substance and the strength of the $\mathrm{H}$ bond formed. However, the dipole approximation of the hydrogen bond may be unsatisfactory because of the large charge separation within the molecule relative to the distance of approach of the second molecule. It is claimed that the charge distribution of the non-bonding electrons of the acceptor atom must be explicitly included in the calculation ${ }^{6,7}$. The hybridization of the bonding orbitals leads to a hybridization also of the non-bonding orbitals, an important consequence of which is that the charge distribution of the orbital relative to the nucleus is made unsymmetrical, giving rise to an effective orbital dipole. Calculation of the electrostatic interaction based on point charges placed at the centroids of charge of the hybridized nonbonding orbitals of the oxygen atom is claimed to give a much more reliable prediction of the strength of the H-bond formed.

If the same arguments are valid for electrostatic interaction with an alkali ion it seems probable that the ether oxygen, in spite of the low dipole moment of the ether, will show a considerable capacity to interact with and solvate the alkali ion.

It is commonly accepted that, as the radius of the $\mathrm{H}$-atom is small, only one $\mathrm{O}$-atom can act as the acceptor atom by the formation of a $\mathrm{H}$-bond. The relatively strong solvation of the $\mathrm{K}^{+}$ion by ethylene glycol dimethyl ether is, however, possibly due to a cooperating effect of two $\mathrm{O}$-atoms on the same molecule:



A similar picture has been drawn by Zook ${ }^{4}$ to explain the increased reactivities of certain enolate ions in ethylene glycol dimethyl ether. The much greater effect of diethylene glycol dimethyl ether may possibly be due to a cooperation of all three oxygen atoms of the same molecule in the solvation of $\mathrm{K}^{+}$. However, it may be that also in the latter case only two oxygen atoms of the same molecule are effective, if the cooperation of the 1,3-oxygen atoms, $-\stackrel{\mathbf{1}}{\mathbf{O}}-\mathbf{R}-\stackrel{\mathbf{2}}{\mathbf{O}}-\mathbf{R}-\stackrel{\mathbf{3}}{\mathbf{O}}-$, is sterically more favourable than that of the $1,2-$ atoms. Again the increased effect of tetraethylene glycol dimethyl ether may be explained by a cooperation of four or five $\mathrm{O}$-atoms on the same molecule 
or by a more favourable sterical position of two or three cooperating oxygen atoms.

\section{FURTHER ISOMERIZATION}

With the ordinary methods of isomerization, only about $50 \%$ of the linolenic acid is converted to the corresponding conjugated tri-ene acid, while the rest remains in a state where only two of the double bonds are conjugated. This is explained by Riemenschneider ${ }^{8}$ by the following reaction scheme:

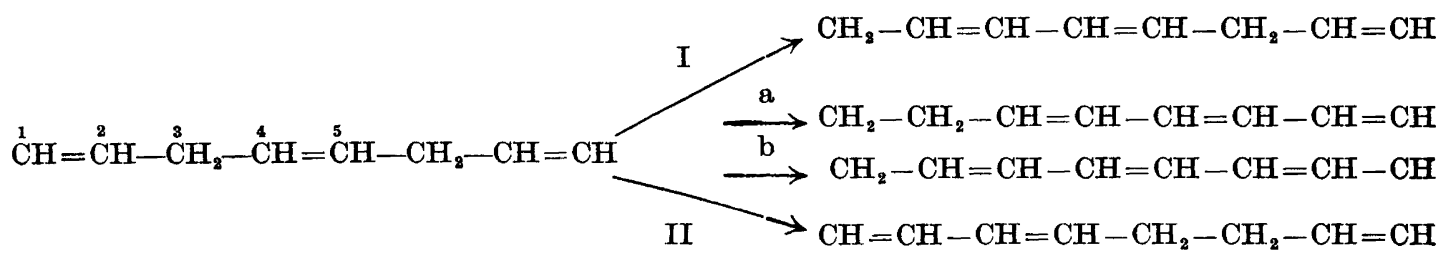

The hydrogen abstracted from C-atom No. 3 will either be transported to C-atom No. 1 or to C-atom No. 5. In both cases the result is an acid with two double bonds in conjugation. In case I the remaining double bond is separated from the conjugated system by a single $\mathrm{CH}_{2}$-group only, and the reaction proceeds rapidly to a complete conjugation by either Scheme $a$ or $b$. In Case II the remaining double bond is separated from the conjugated system by two $\mathrm{CH}_{2}$-groups, and any further reaction will be expected to be much slower. Preliminary studies with prolonged reaction times using very active catalyst solutions seem to indicate that a further conjugation isomerization occurs, bringing about a more complete conjugation isomerization of the linolenic acid. Some of these results are given in Table 4. Sreenivasan and Brown obtained approximately the same degree of conjugation of the linolenic acid after $2 \mathrm{~h}$ at $140^{\circ} \mathrm{C}$ using $t$-butanol as solvent ${ }^{9}$.

Table 4. Extinction coefficient at $267 \mathrm{~m} \mu$ and $234 \mathrm{~m} \mu$ at prolonged reaction times. Catalyst solution: $10 \mathrm{ml}$, containing 0.4 mole/l $\mathrm{K}$-t-butylate. Temp. $25^{\circ} \mathrm{C}$.

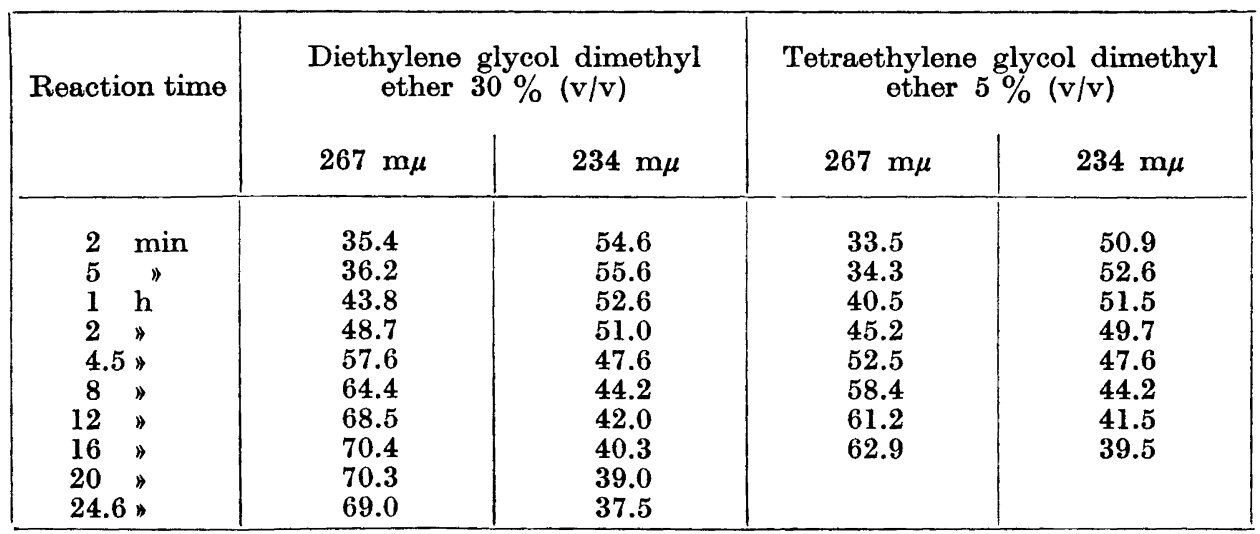

Acta Chem. Scand. 17 (1963) No. 1 


\section{REFERENCES}

1. Ugelstad, J., Jenssen, B. and Mörk, P. C. Acta Chem. Scand. 16 (1962) 323.

2a. Zaugg, H. E. J. Am. Chem. Soc. 82 (1960) 2903.

2b. Zaugg, H. E. J. Am. Chem. Soc. 83 (1961) 837.

3. Scott, N. D., Walker, J. F. and Hawsley, V. L. J. Am. Chem. Soc. 58 (1936) 2442.

4. Zook, H. D. and Russo, T. I. J. Am. Chem. Soc. 82 (1960) 1258.

5. Sreenivasan, B. S. and Brown, J. B. J. Am. Oil Chemists' Soc. 33 (1956) 521.

6. Lennard-Jones, J. and Pople, J. A. Proc. Roy. Soc. (London) 205A (1951) 155.

7. Schneider, W. G. J. Chem. Phys. 23 (1955) 26.

8. Riemenschneider, R. W. J. Am. Oil Chemists' Soc. 31 (1954) 517.

9. Sreenivasan, B. S. and Brown, J. B. J. Am. Oil Chemists' Soc. 35 (1958) 89.

Received August 28, 1962. 